\title{
Thermally Robust Zirconia Nanorod/Polyimide Hybrid Films as a Highly Flexible Dielectric Material
}

Yong-Zhu Yan a, Sung Soo Park a, Ha Ram Moon a, Wei-Jin Zhang a, Shuai Yuan b,c,*, Liyi Shi b,c, Dong Gi Seong a, and Chang-Sik Ha ${ }^{a}$,*

aDepartment of Polymer Science and Engineering, School of Chemical Engineering, Pusan National University, Busan 46241, Republic of Korea

bResearch Center of Nanoscience and Nanotechnology, Shanghai University, Shanghai 200444, China.

cEmerging Industries Institute, Shanghai University, Jiaxing, Zhejiang 314006, China

Corresponding Authors

* Chang-Sik Ha E-mail: csha@pnu.edu

* Shuai Yuan E-mail: s.yuan@shu.edu.cn 


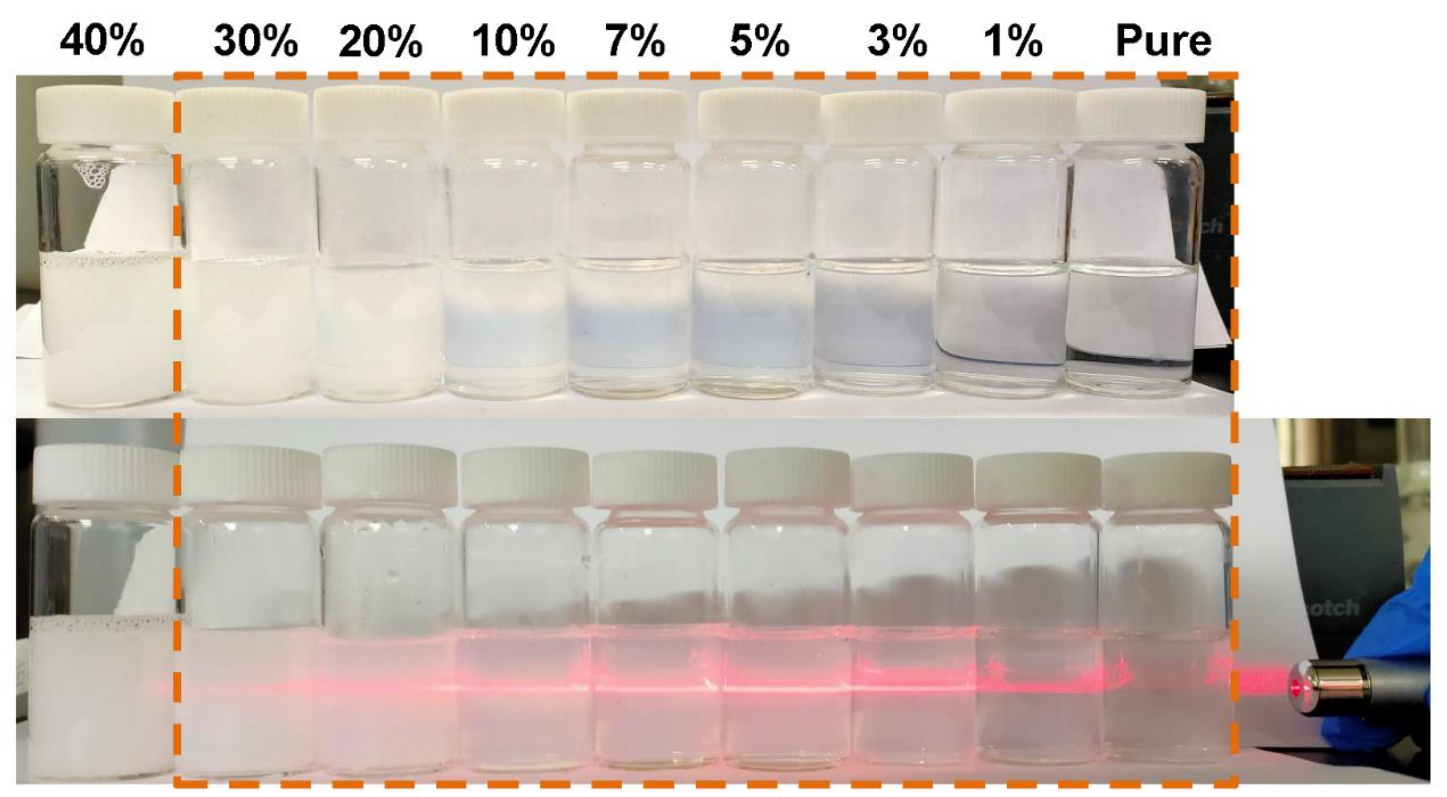

Figure S1. Optical images of $\mathrm{ZrO}_{2}$ nanorods dispersed in water with different amounts. 


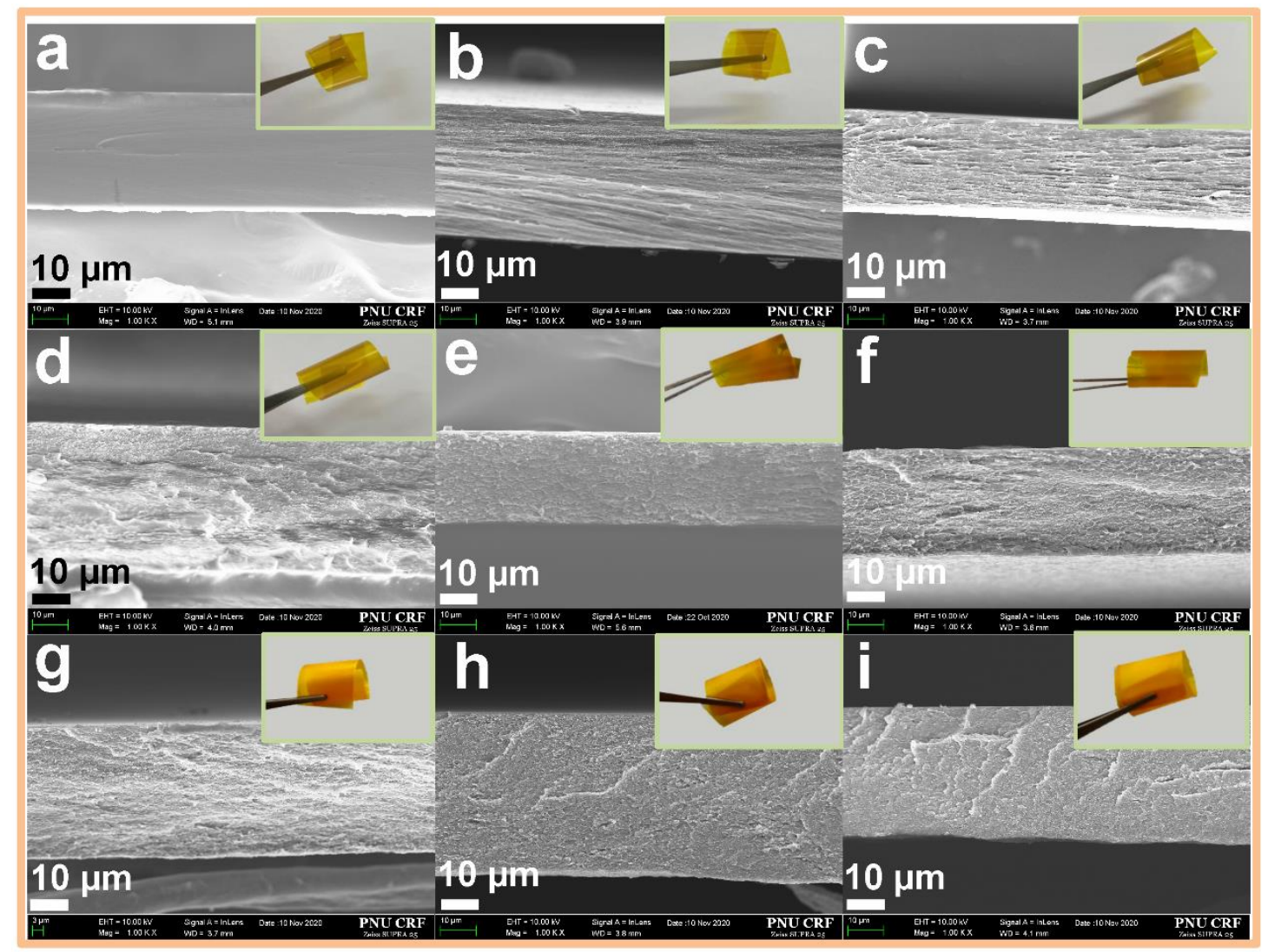

Figure S2. Low resolution fractured cross-sectional SEM images of (a) pristine PI, (b)

1\%-ZrO 2 -PI, (c) $3 \%-\mathrm{ZrO}_{2}-\mathrm{PI}$, (d) $5 \%-\mathrm{ZrO}_{2}-\mathrm{PI}$, (e) $7 \%-\mathrm{ZrO}_{2}-\mathrm{PI}$, (f) $10 \%-\mathrm{ZrO}_{2}-\mathrm{PI}$, (g)

20\%-ZrO2-PI, (h) 30\%-ZrO2-PI, (i) 40\%-ZrO 2 -PI. 

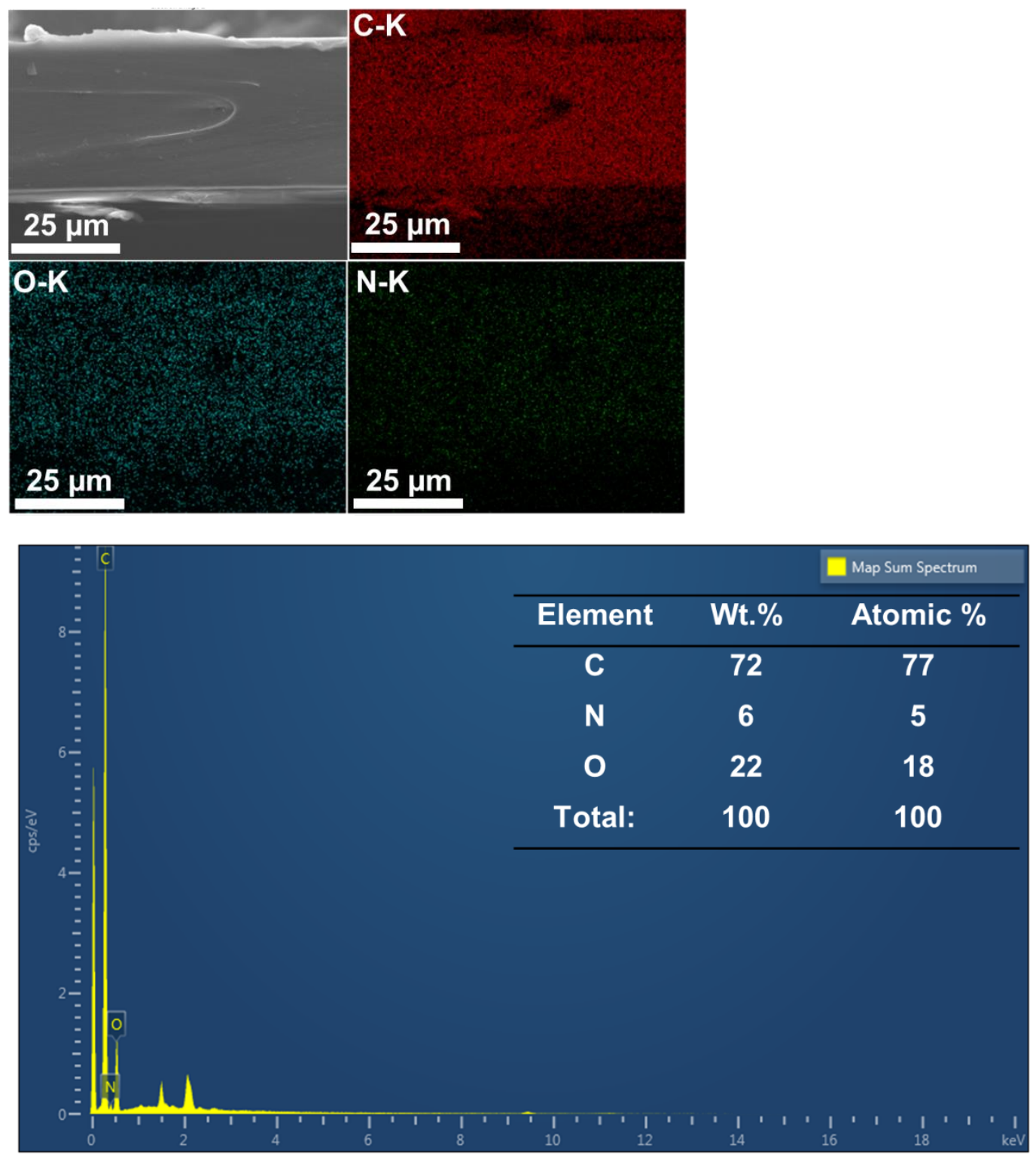

Figure S3. SEM-EDX mapping micrographs of pristine PI. 

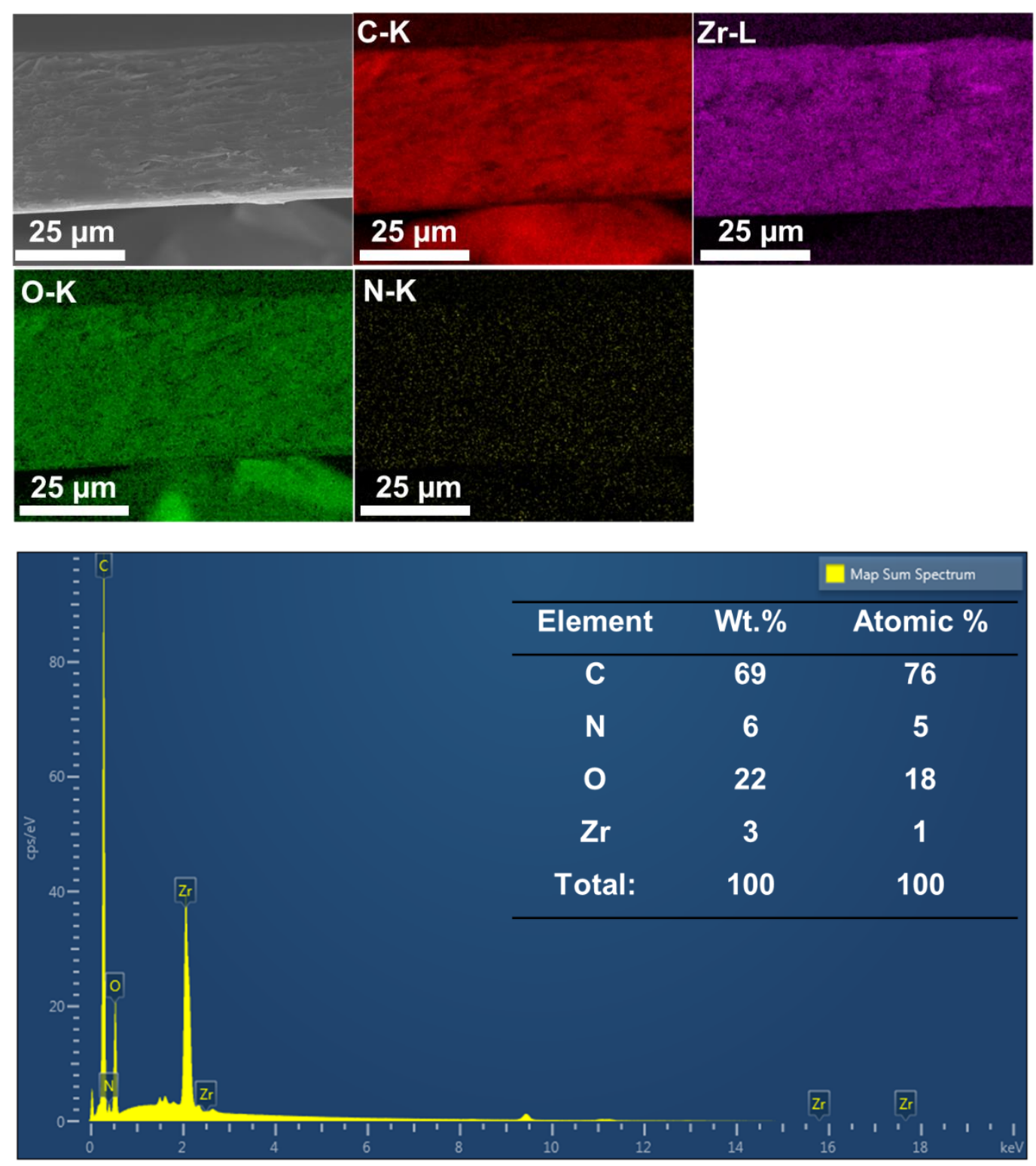

*[Note: Since the Atomic \% value of the $\mathrm{Zr}$ element of this sample is small, we also provide data with one decimal place: $\operatorname{Zr}(0.5$ Atomic \%).]

Figure S4. SEM-EDX mapping micrographs of 3\%- $\mathrm{ZrO}_{2}-\mathrm{PI}$. 

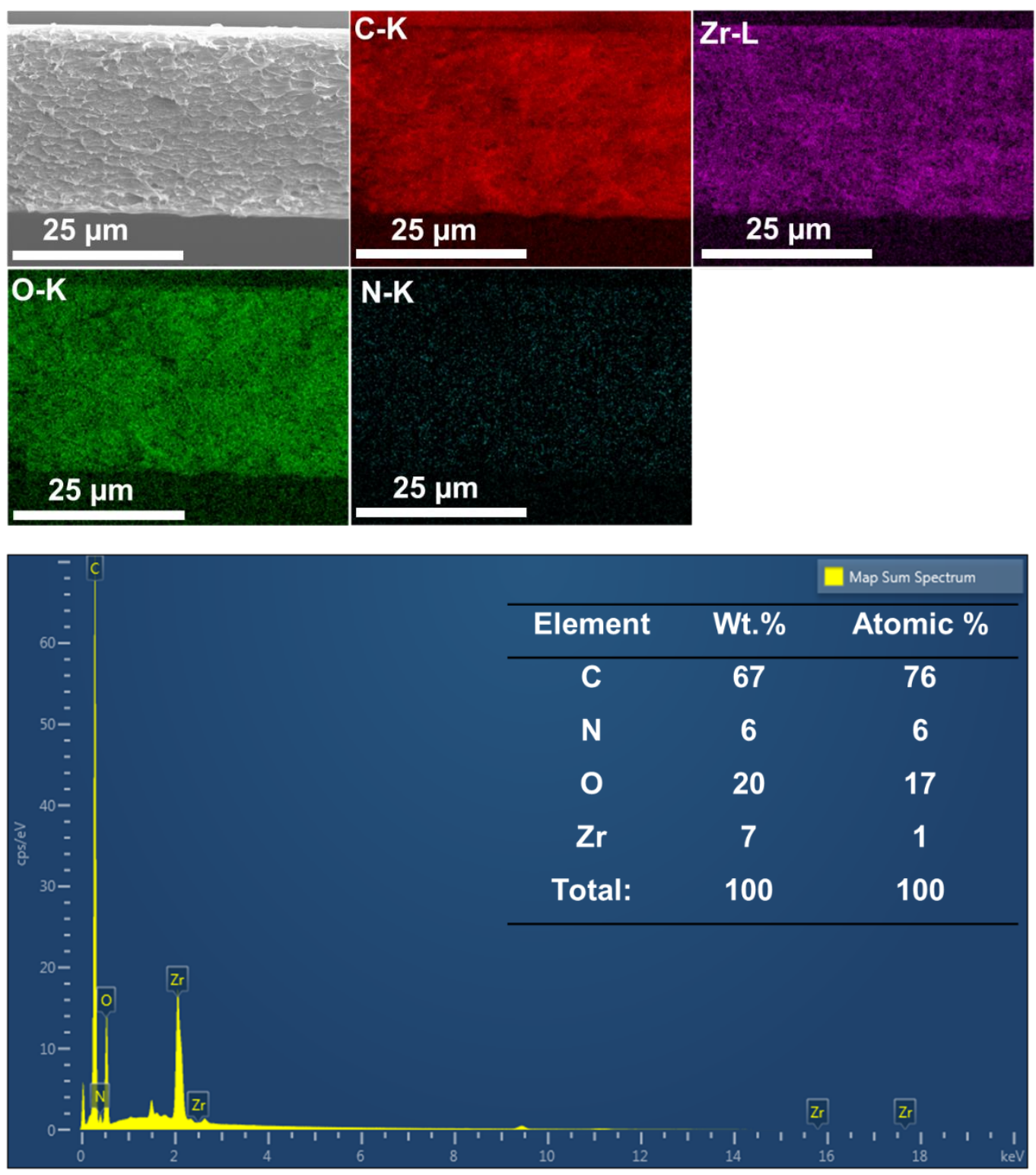

*[Note: Since the Atomic \% value of the $\mathrm{Zr}$ element of this sample is small, we also provide data with one decimal place: $\operatorname{Zr}(1.1$ Atomic \%).]

Figure S5. SEM-EDX mapping micrographs of 7\%- $\mathrm{ZrO}_{2}-\mathrm{PI}$. 

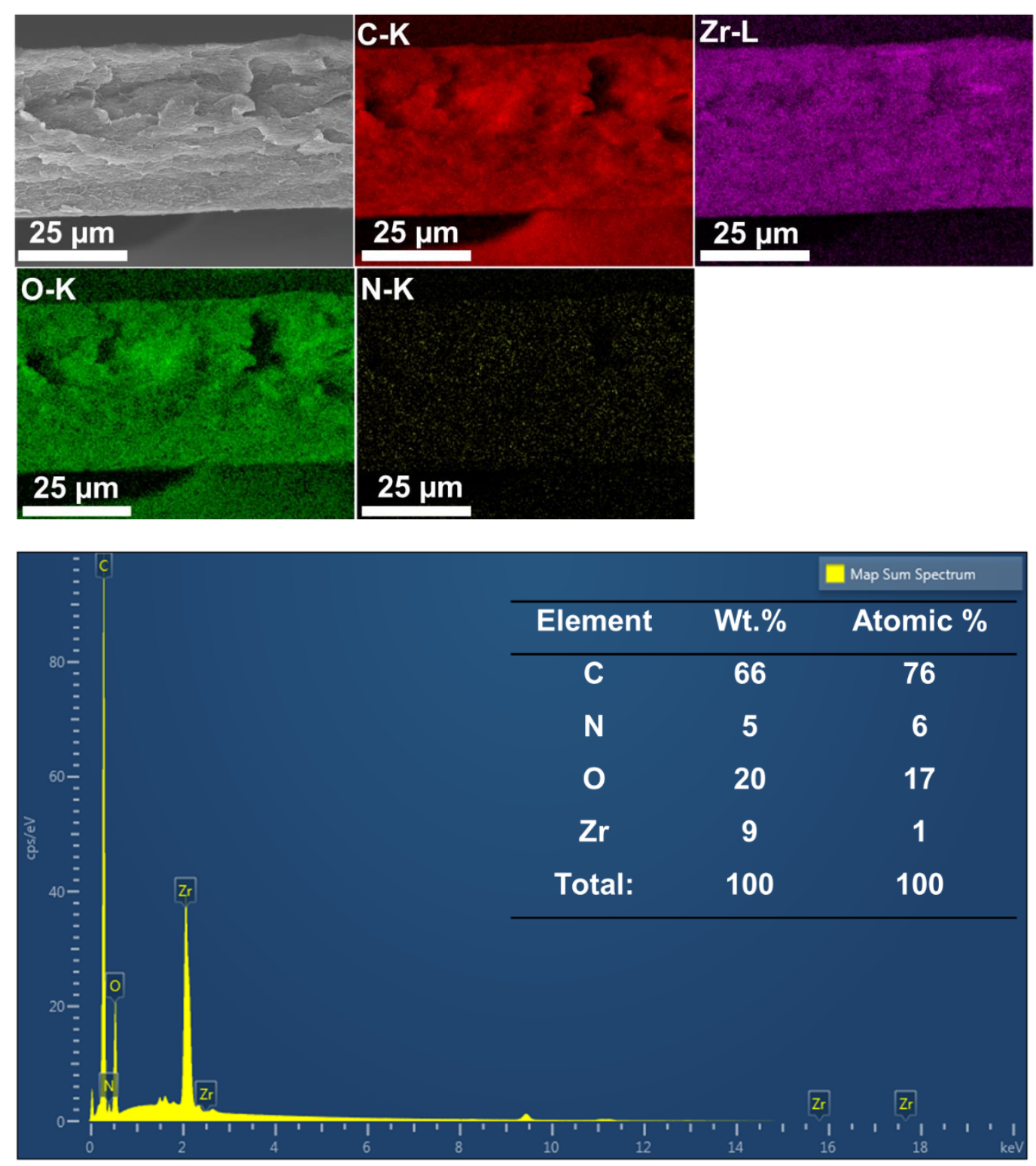

*[Note: Since the Atomic \% value of the $\mathrm{Zr}$ element of this sample is small, we also provide data with one decimal place: $\operatorname{Zr}(1.3$ Atomic \%).]

Figure S6. SEM-EDX mapping micrographs of $10 \%-\mathrm{ZrO}_{2}-\mathrm{PI}$. 

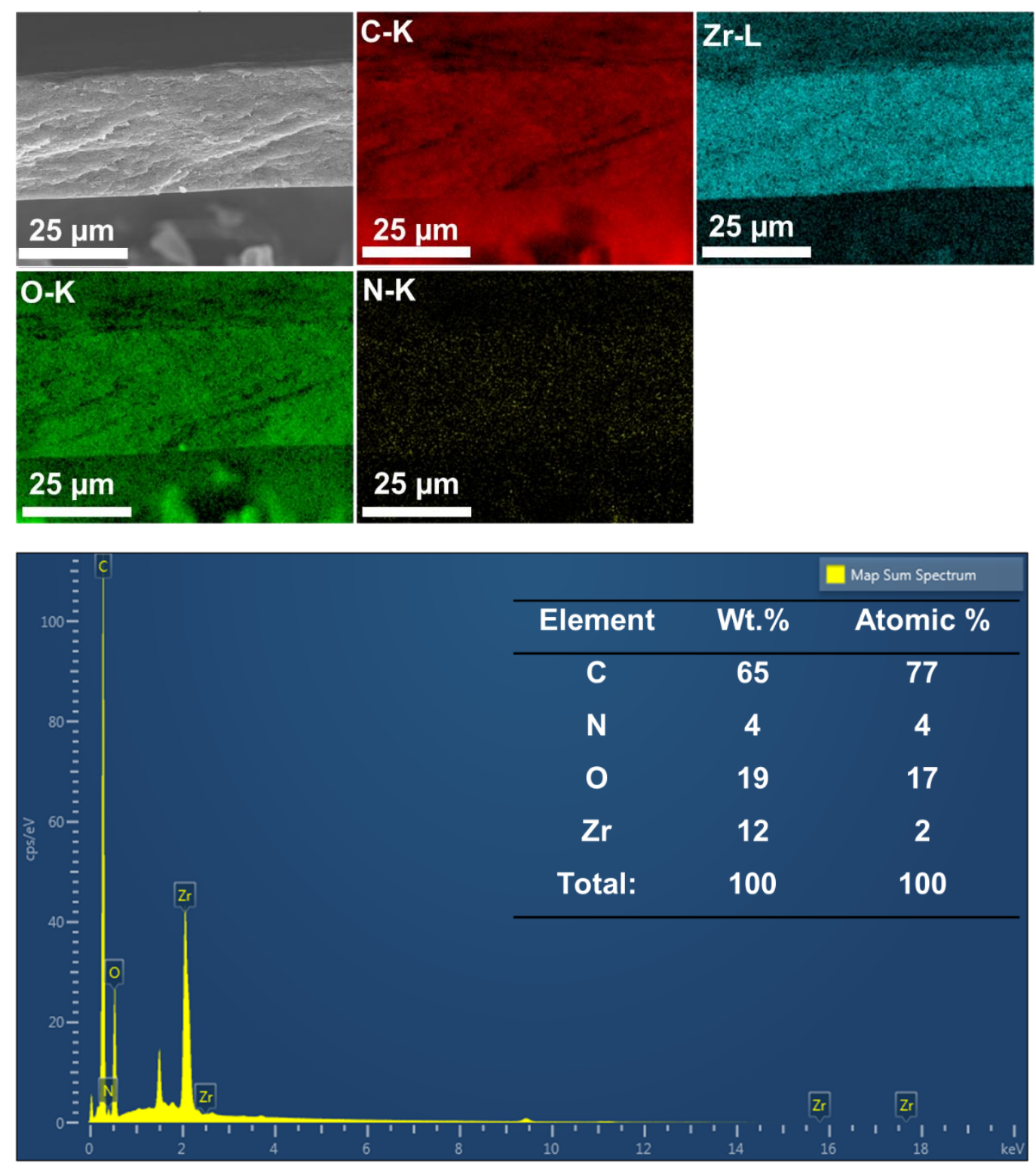

Figure S7. SEM-EDX mapping micrographs of $20 \%-\mathrm{ZrO}_{2}-\mathrm{PI}$. 

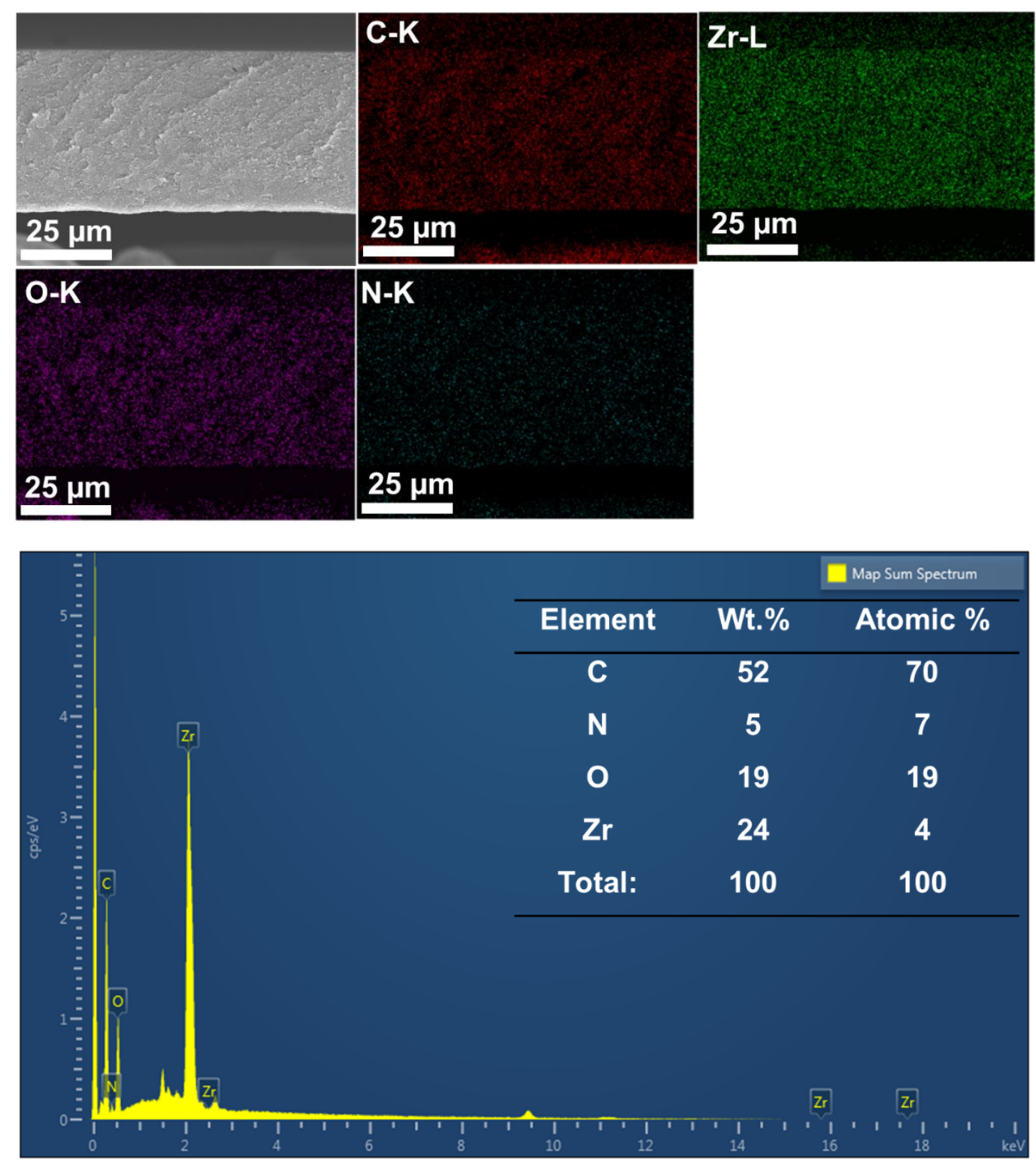

Figure S8. SEM-EDX mapping micrographs of $30 \%-\mathrm{ZrO}_{2}-\mathrm{PI}$. 


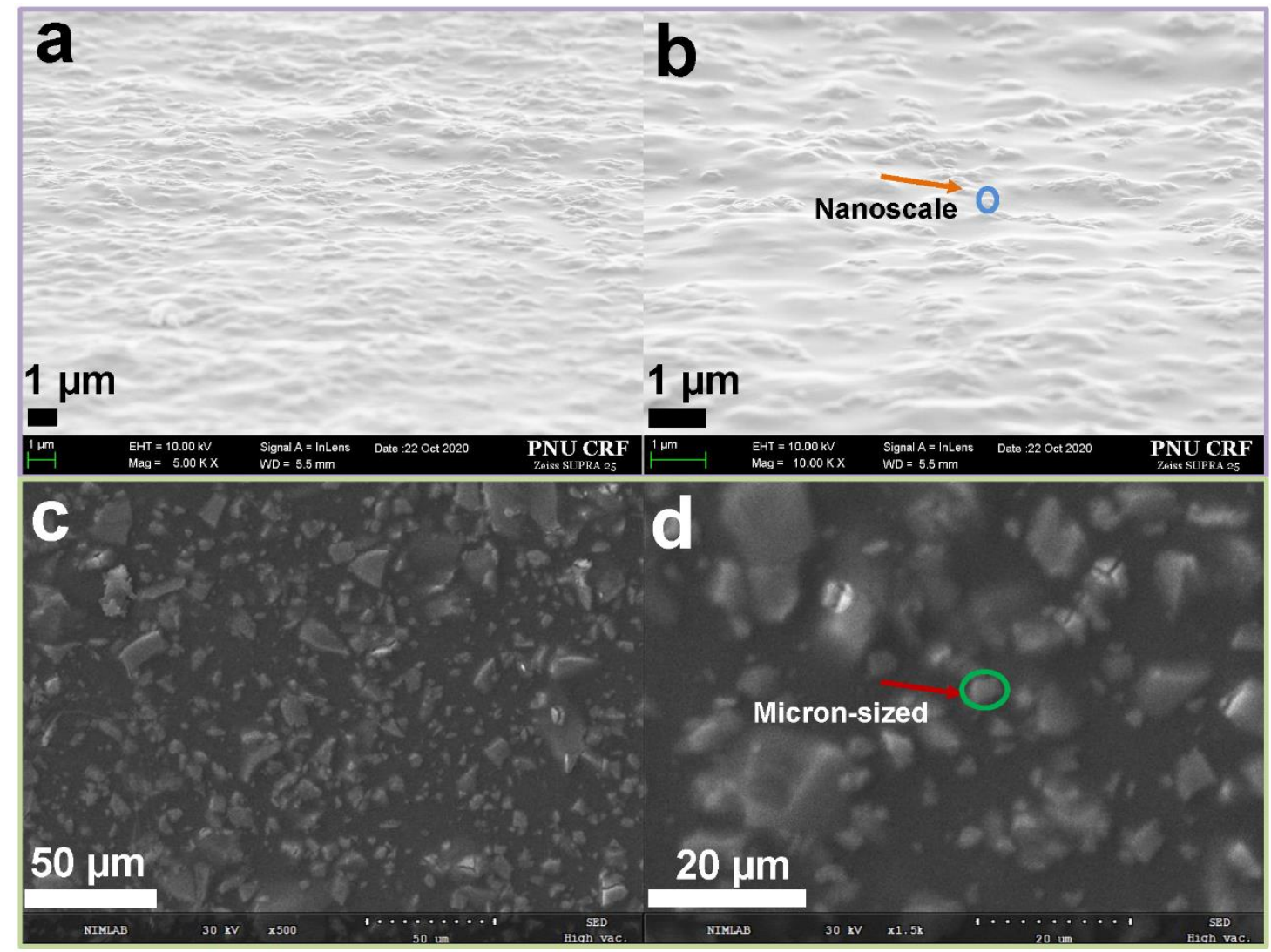

Figure S9. (a and b) Surface SEM images of 7\%- $\mathrm{ZrO}_{2}-\mathrm{PI}$, and (c and d) $7 \%-\mathrm{ZrO}_{2}-$ PIDMAc, where the $\mathrm{ZrO}_{2}-\mathrm{PI}_{\text {DMAc }}$ nanohybrid film was synthesized using DMAc as a solvent. 


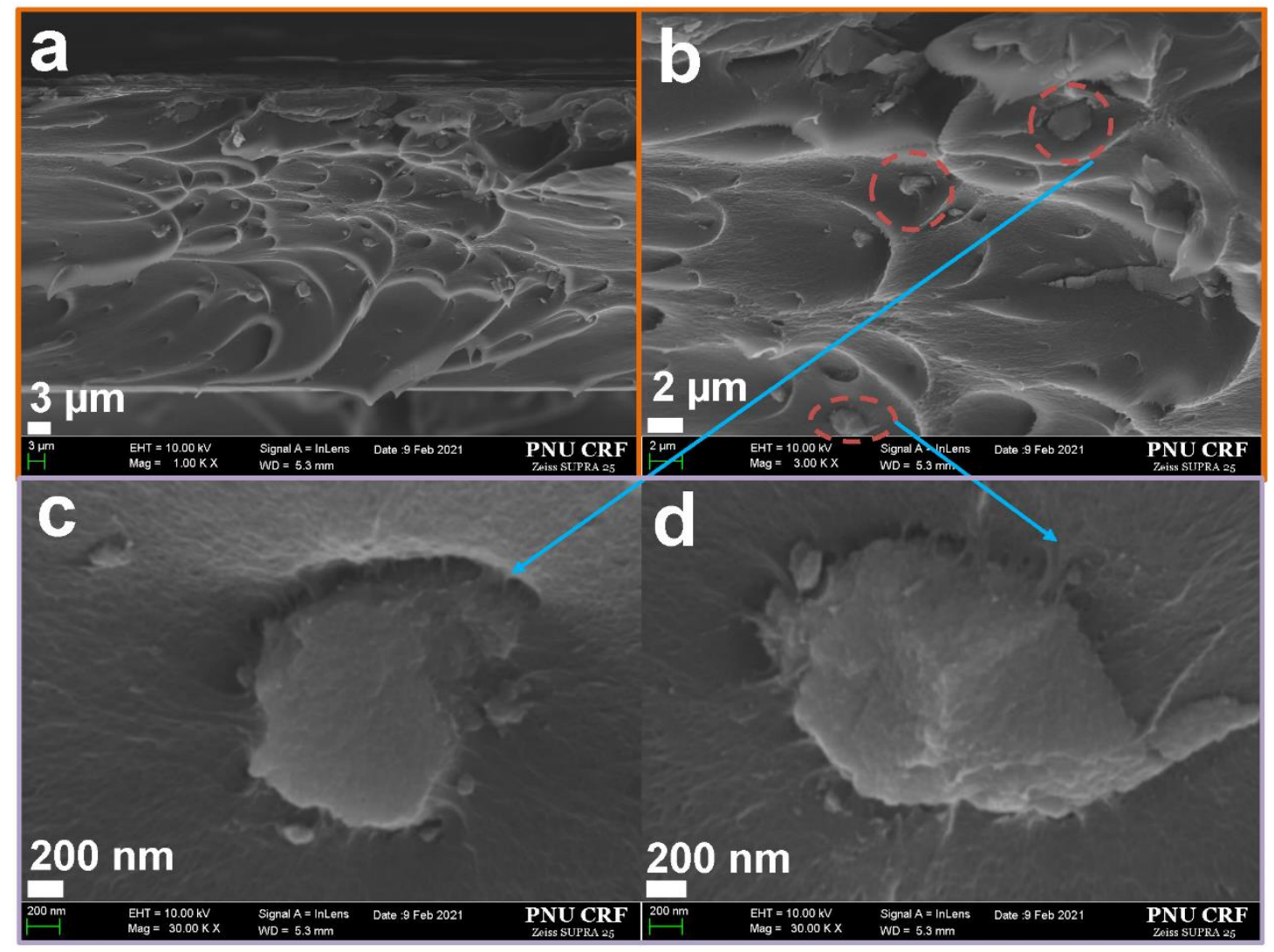

Figure S10. ( $a$ and b) Low resolution fractured cross-sectional SEM images of 7\%$\mathrm{ZrO}_{2}-\mathrm{PI}_{\mathrm{DMAc}}$, (c and d) High resolution fractured cross-sectional SEM images of 7\%$\mathrm{ZrO}_{2}-\mathrm{PI}_{\mathrm{DMAc}}$, where $\mathrm{ZrO}_{2}$ nanorod aggregates existing in the PI matrix are marked with red circles. 


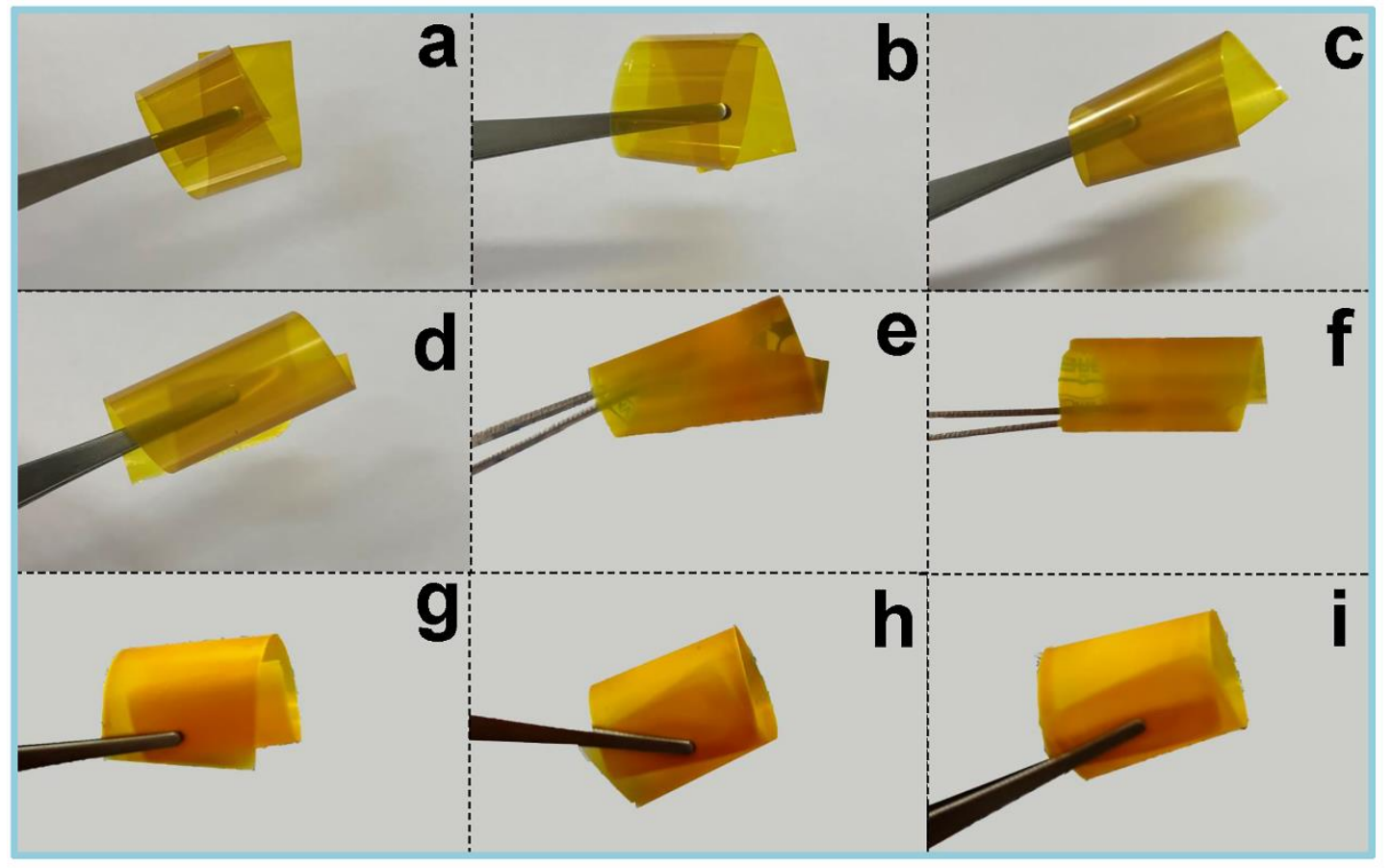

Figure S11. Photographs of (a) pristine PI, (b) $1 \%-\mathrm{ZrO}_{2}-\mathrm{PI}$, (c) $3 \%-\mathrm{ZrO}_{2}-\mathrm{PI}$, (d) $5 \%$ $\mathrm{ZrO}_{2}-\mathrm{PI}$, (e) $7 \%-\mathrm{ZrO}_{2}-\mathrm{PI}$, (f) $10 \%-\mathrm{ZrO}_{2}-\mathrm{PI}$, (g) $20 \%-\mathrm{ZrO}_{2}-\mathrm{PI}$, (h) $30 \%-\mathrm{ZrO}_{2}-\mathrm{PI}$, (i) $40 \%-\mathrm{ZrO}_{2}$-PI. 


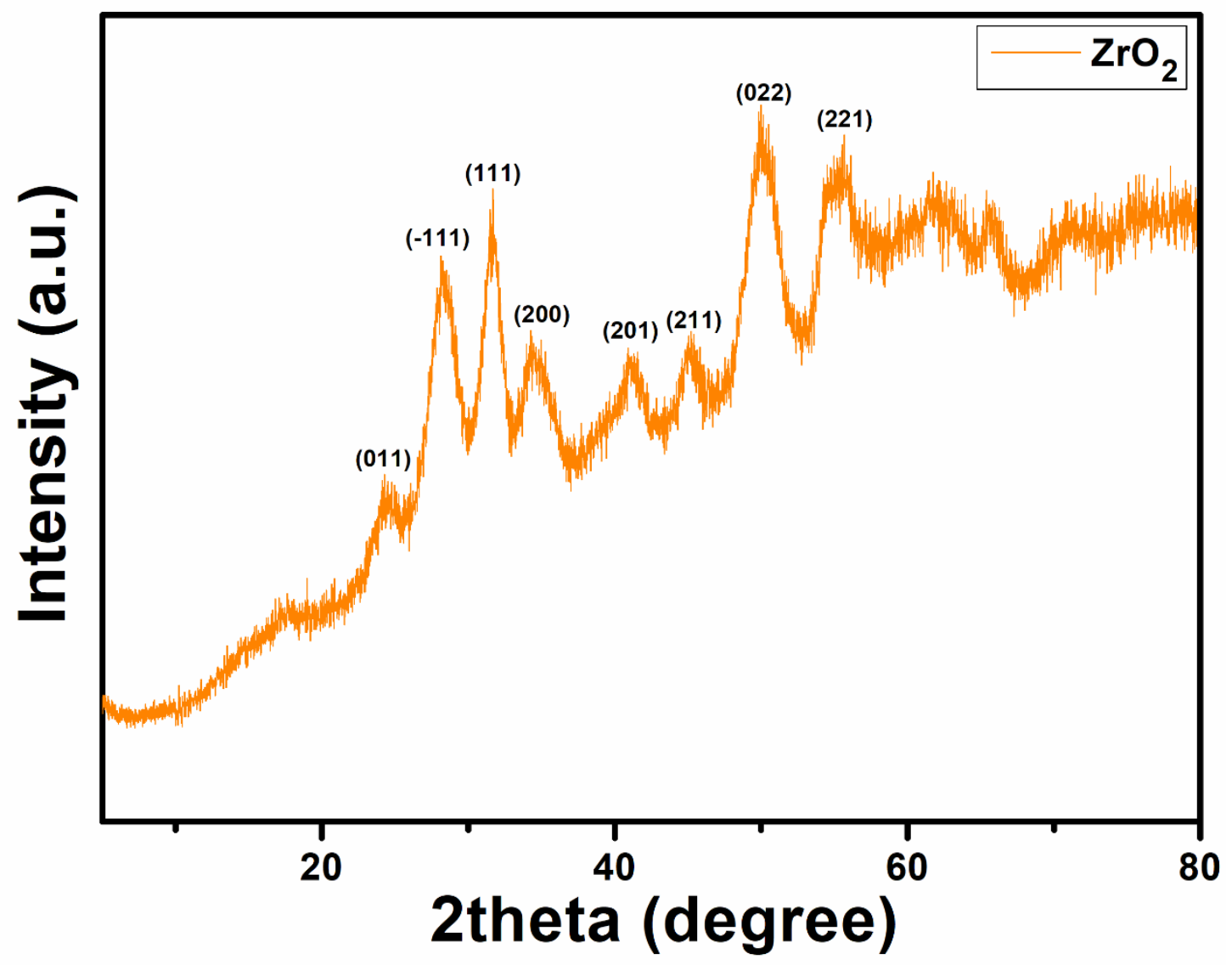

Figure S12. XRD pattern of $\mathrm{ZrO}_{2}$ nanorods. 


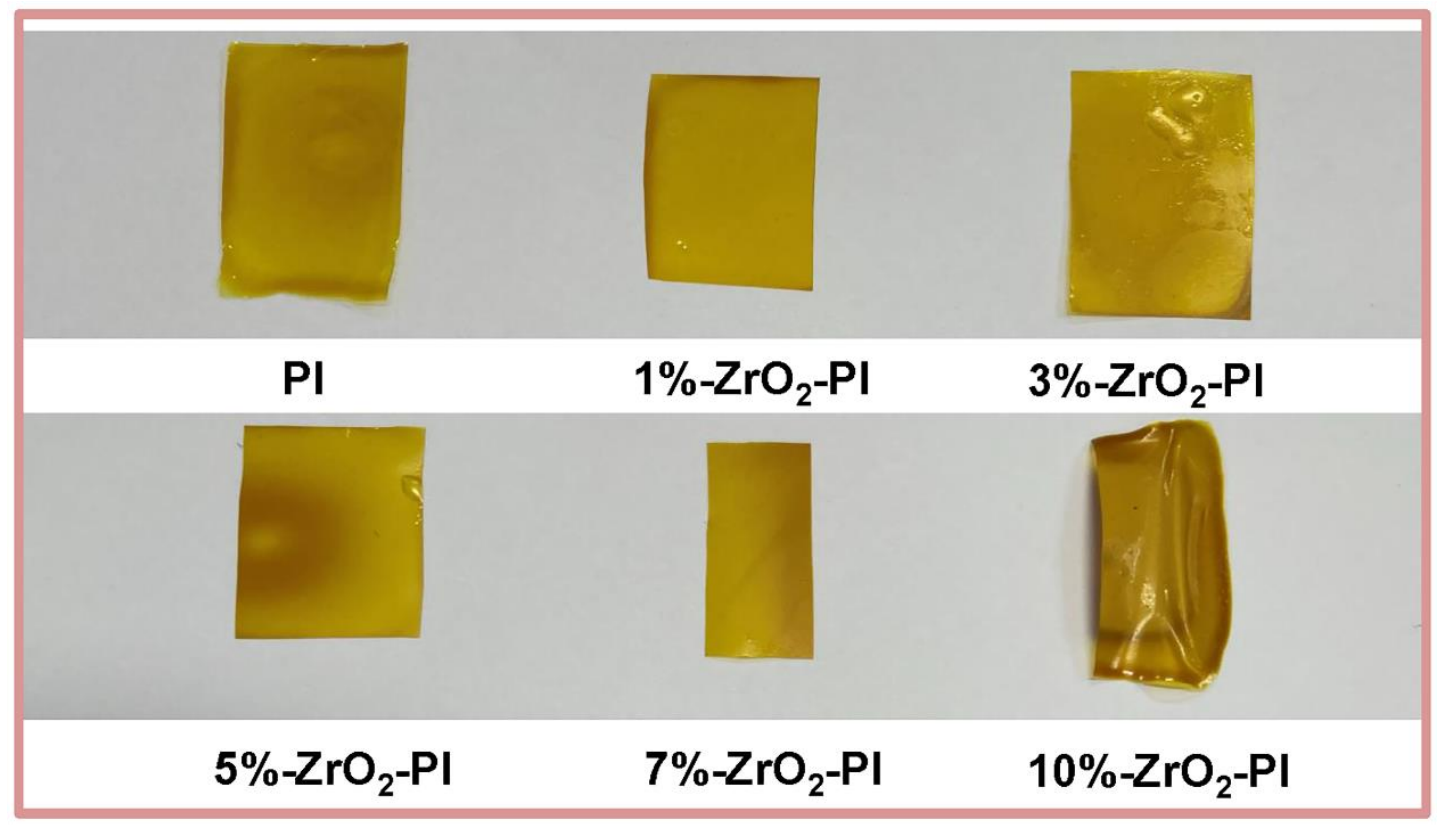

Figure S13. Photographs of neat PI and $\mathrm{ZrO}_{2}-\mathrm{PI}$ nanohybrid films. (derived from PAA solution after 3 months of storage) 


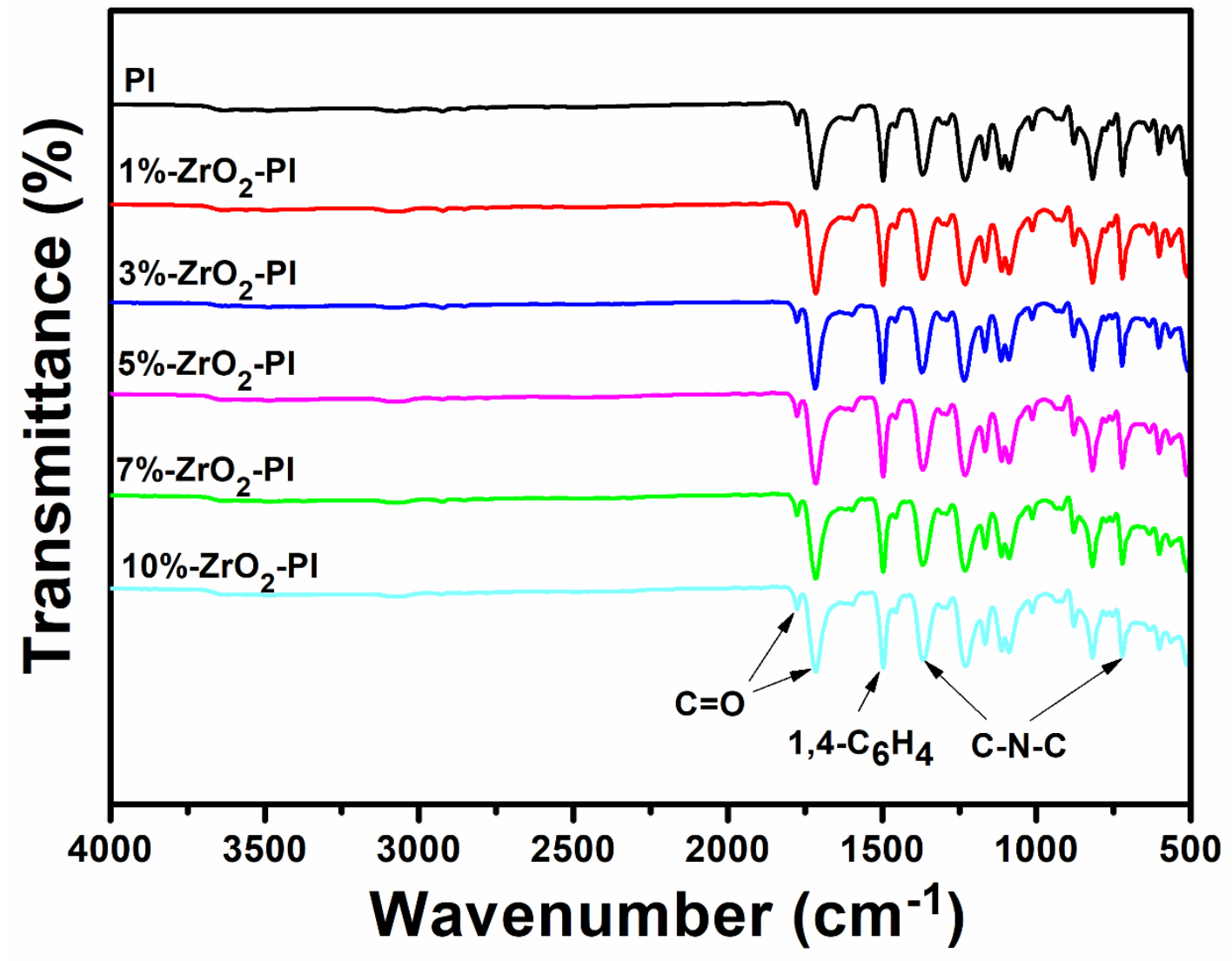

Figure S14. FT-IR spectra of neat PI and $\mathrm{ZrO}_{2}-\mathrm{PI}$ nanohybrid films. (derived from PAA solution after 3 months of storage) 
Table S1. The thermal stability and CTE values of $\mathrm{ZrO}_{2}$-PI nanohybrid films.

\begin{tabular}{|c|c|c|c|c|}
\hline Sample & $\mathrm{T}_{\mathrm{d}}{ }^{5}\left({ }^{\circ} \mathrm{C}\right)^{\mathrm{a}}$ & $\mathrm{T}_{\mathrm{d}}{ }^{10}\left({ }^{\circ} \mathrm{C}\right)^{\mathrm{a}}$ & $\mathrm{T}^{30}\left({ }^{\circ} \mathrm{C}\right)^{\mathrm{a}}$ & CTE $\left(\mathrm{ppm} \mathrm{K}^{-1}\right)$ \\
\hline PI & 567 & 586 & 626 & 22.2 \\
\hline $1 \%-\mathrm{ZrO}_{2}-\mathrm{PI}$ & 561 & 581 & 625 & 21.2 \\
\hline $3 \%-\mathrm{ZrO}_{2}-\mathrm{PI}$ & 555 & 580 & 624 & 21.3 \\
\hline $5 \%-\mathrm{ZrO}_{2}-\mathrm{PI}$ & 552 & 584 & 633 & 22.1 \\
\hline $7 \%-\mathrm{ZrO}_{2}-\mathrm{PI}$ & 552 & 588 & 638 & 17.9 \\
\hline $10 \%-\mathrm{ZrO}_{2}-\mathrm{PI}$ & 549 & 589 & 647 & 17.8 \\
\hline $20 \%-\mathrm{ZrO}_{2}-\mathrm{PI}$ & 528 & 588 & 678 & 16.6 \\
\hline $30 \%-\mathrm{ZrO}_{2}-\mathrm{PI}$ & 502 & 586 & 705 & 16.3 \\
\hline $40 \%-\mathrm{ZrO}_{2}-\mathrm{PI}$ & 516 & 601 & 773 & 17.0 \\
\hline
\end{tabular}

${ }^{\mathrm{a}} \mathrm{T}_{\mathrm{d}}{ }^{5}\left({ }^{\circ} \mathrm{C}\right), \mathrm{T}_{\mathrm{d}}{ }^{10}\left({ }^{\circ} \mathrm{C}\right)$ and $\mathrm{T}_{\mathrm{d}}{ }^{30}\left({ }^{\circ} \mathrm{C}\right)^{\mathrm{a}}$ are the temperatures at which $5 \mathrm{wt} . \%, 10 \mathrm{wt} . \%$ and $30 \mathrm{wt} . \%$ of loss, respectively, take place. 
Table S2. Comparison of thermal properties (CTE and $\mathrm{T}_{\mathrm{g}}$ ) values of $\mathrm{ZrO}_{2}-\mathrm{PI}$ nanohybrid films with those of other reported polyimide-based hybrid films.

\begin{tabular}{|c|c|c|c|c|}
\hline Samples* & $\begin{array}{l}\text { Filler Content } \\
\text { (Range) }\end{array}$ & $\operatorname{Tg}\left({ }^{\circ} \mathrm{C}\right)$ & CTE $\left(p p m K^{-1}\right)$ & References \\
\hline $\mathrm{PI} \mathrm{ZrO}_{2}$ hybrid films & $0-15$ wt. $\%$ & $>400$ & $N A$ & {$[27]$} \\
\hline $\mathrm{PI} / \mathrm{MS}$ & $0-10$ wt. $\%$ & $352-368$ & $N A$ & {$[36]$} \\
\hline CCTO-OH/(PI) & $0-40$ wt. $\%$ & $372-390$ & $17-51$ & {$[45]$} \\
\hline $\mathrm{ZrW}_{2} \mathrm{O}_{8} / \mathrm{BTDA}-\mathrm{ODA}$ & $0-50$ wt. $\%$ & $274-287$ & $23-33$ & [48] \\
\hline API/nano-silica & $0-30$ wt. $\%$ & $292-328$ & $16.6-20.5$ & [49] \\
\hline $\mathrm{PI} / \mathrm{MMT}$ & $0-5$ wt. $\%$ & $289.8-305.1$ & $28-46$ & {$[50]$} \\
\hline Polyimide $/ \mathrm{ZrW}_{2} \mathrm{O}_{8}$ & $0-50$ wt. $\%$ & $N A$ & $31-45$ & {$[51]$} \\
\hline $\mathrm{SiO}_{2}-\mathrm{PI}$ & $0-20$ wt. $\%$ & $363.3-381.3$ & $27.8-33.9$ & {$[52]$} \\
\hline $\mathrm{PI} / \mathrm{SiC}$ nanofibers net & $0-25$ wt. $\%$ & $350.7-364.5$ & $9.7-35.9$ & {$[53]$} \\
\hline PHI-6FZr & $0-50$ wt. $\%$ & $350-410$ & $25-73$ & {$[54]$} \\
\hline $\mathrm{PI} @ \mathrm{BT} / \mathrm{PI}$ & $0-55$ wt. $\%$ & $385-389$ & $N A$ & {$[\mathrm{~S} 1]$} \\
\hline GFO/pPI & $0-1$ wt. $\%$ & $355-388$ & $24.9-48.7$ & {$[\mathrm{~S} 2]$} \\
\hline $\mathrm{PI} / \mathrm{f}-\mathrm{BN} / \mathrm{g}-\operatorname{TrG}$ & $0-50$ wt. $\%$ & $403-419$ & $10-45$ & [S3] \\
\hline 4ATA-PI/ZrO 2 & $0-50$ wt. $\%$ & $387-410$ & $2.93-37$ & {$[\mathrm{~S} 4]$} \\
\hline $3 \mathrm{SOH}-\mathrm{RPI} / \mathrm{ZrO}_{2}$ & $0-30$ wt. $\%$ & $230-287$ & $52-72$ & {$[\mathrm{~S} 5]$} \\
\hline PI/BN Composites & $0-30$ wt. $\%$ & $368-382$ & $27-44$ & [S6] \\
\hline $\begin{array}{l}\mathrm{ZrO}_{2} \text {-PI films derived from } \\
\text { water-soluble PAS }\end{array}$ & $0-40$ wt. $\%$ & $394.6-404.7$ & $11.8-18.9$ & This work \\
\hline
\end{tabular}

*[Note: $N A$ represents that this property data is not available; A brief explanation of the above polyimide based hybrid films is summarized as follows].

${ }^{27} \mathrm{PI}-\mathrm{ZrO}_{2}$ hybrid films: 2,2-Bis(3-amino-4-hydroxyphenyl) hexafluoropropane / 4,4-oxydiphthalic anhydride) polyimide /nanocrystalline-zirconium dioxide hybrid materials;

${ }^{36} \mathrm{PI} / \mathrm{MS}$ : Polyimide/mesoporous silica hybrid films based on water-soluble poly(amic acid) ammonium salt;

${ }^{45} \mathrm{CCTO}-\mathrm{OH} /(\mathrm{PI})$ : Surface hydroxylated $\mathrm{CaCu}_{3} \mathrm{Ti}_{4} \mathrm{O}_{12}(\mathrm{CCTO}-\mathrm{OH}) /$ poly$($ pyromellitic dianhydride-co-4,4'oxydianiline)(PMDA-ODA PI);

${ }^{48} \mathrm{ZrW}_{2} \mathrm{O}_{8} / \mathrm{BTDA}-\mathrm{ODA}: \mathrm{ZrW}_{2} \mathrm{O}_{8}$ powders surface-derivatized with (3-aminopropyl)siloxy linker molecules, dispersed in 3,3',4,4'-biphenyltetracarboxylic dianhydride (BPDA)- 4,4'-oxydianiline (ODA) polyamic acid (PAA) resins to give thermally cured $\mathrm{ZrW}_{2} \mathrm{O}_{8} / \mathrm{PI}$ hybrid;

${ }^{49} \mathrm{API} /$ nano-silica: Functionalized hybrid film from API (9,10-bis(4-aminopheny)adenine ( $p$-APA) and 3,3',4,4'tetracarboxydiphthalic ether dianhydride (ODPA)) with nano-silica;

${ }^{50} \mathrm{PI} / \mathrm{MMT}$ : PI/montmorillonite nanocomposites;

${ }^{51}$ Polyimide/ $\mathrm{ZrW}_{2} \mathrm{O}_{8}$ : BTDA-ODA PAA to form $\mathrm{PI} / \mathrm{ZrW}_{2} \mathrm{O}_{8}$ hybrids;

${ }^{52} \mathrm{SiO}_{2}-\mathrm{PI}$ : (3-trimethoxysilylpropyl)diethylenetriamine- $\mathrm{SiO}_{2} / \mathrm{PI}$; 
${ }^{53} \mathrm{PI} / \mathrm{SiC}$ nanofibers net: Embedding the partial imidized PI/SiC nanofiber-net in a PAA solution, followed by removing the solvent and imidization of the PAA;

${ }^{54} \mathrm{PHI}-6 \mathrm{FZr}$ : Hydroxyl-containing polyimides PHIs (DHTM with 6FDA in $m$-cresol)/ZrO 2 ;

S1PI@BT/PI: Core-shell structured polyimide@ $\mathrm{BaTiO}_{3}(\mathrm{PI} @ \mathrm{BT})$ nanoparticles that were fabricated via in-situ polymerization of poly(amic acid) (PAA) and the following thermal imidization;

${ }^{\mathrm{S} 2} \mathrm{GFO} / \mathrm{pPI}$ : Porous PEG-graphene fluoroxide/polyimide;

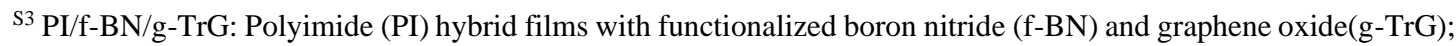

S44ATA-PI/ZrO2: Bio-based polyimide (4ATA-PI) and the corresponding PI hybrids of $\mathrm{ZrO}_{2}$;

S53SOH-RPI/ZrO 2 : Sulfur-containing poly(o-hydroxy-imide)s (3SOH-RPI) with pendant hydroxyl groups and the corresponding $3 \mathrm{SOH}-\mathrm{RPI} / \mathrm{ZrO}_{2}$ polyimide;

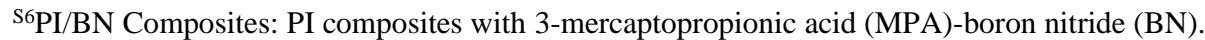


Table S3. Comparison of permittivity, dielectric loss and thermal properties of $10 \%$ $\mathrm{ZrO}_{2}$-PI nanohybrids with other polymer-based hybrids.

\begin{tabular}{llllll}
\hline Samples* & Permittivity & $\begin{array}{l}\text { Dielectric } \\
\text { loss }\end{array}$ & $\mathrm{Td}_{\mathrm{d}}{ }^{\circ}\left({ }^{\circ} \mathrm{C}\right)$ & $\mathrm{Td}^{10}\left({ }^{\circ} \mathrm{C}\right)$ & References \\
\hline rGO/PAN & 40 & 0.26 & $N A$ & $N A$ & {$[11]$} \\
MBHSt & 10 & $N A$ & 246 & $N A$ & {$[14]$} \\
PMMA/BaTiO 3 nanocomposites & 14.6 & 0.037 & $\approx 370$ & $\approx 380$ & {$[\mathrm{~S} 7]$} \\
PS/EG & 37.9 & 1.5 & $N A$ & 384 & {$[\mathrm{~S} 8]$} \\
rGO-PVA/PVDF & 230 & $N A$ & $\mathrm{NA}$ & $\approx 330$ & {$[\mathrm{~S} 9]$} \\
$\mathrm{PVA} / \mathrm{V}_{2} \mathrm{C}$ MXene nanocomposite & 24 & 0.14 & $N A$ & $N A$ & {$[\mathrm{~S} 10]$} \\
$\mathrm{TiO}_{2} / \mathrm{PVP}$ & 5.5 & $N A$ & $N A$ & $N A$ & {$[\mathrm{~S} 11]$} \\
$10 \%-Z r \mathrm{Z}_{2}-\mathrm{PI}$ & 5.1 & 0.047 & 549 & 589 & This work \\
\hline
\end{tabular}

*[Note: $\mathrm{T}_{\mathrm{d}}{ }^{5}\left({ }^{\circ} \mathrm{C}\right)$ and $\mathrm{T}_{\mathrm{d}}{ }^{10}\left({ }^{\circ} \mathrm{C}\right)$ represent the temperatures at which 5 wt. $\%$ and $10 \mathrm{wt} . \%$ of loss was observed, respectively. $N A$ represent that these properties are not available; A brief explanation of the above samples is summarized as follows].

${ }^{11}$ rGO/PAN: Polyacrylonitrile (PAN) nanofiber mats containing graphene oxide (GO) with core-shell microstructure;

${ }^{14}$ MBHSt: Copolymers of acrylic monomers (methyl methacrylate (MMA), butyl acrylate (BA), mono(2acryloyloxyethyl) succinate (HEA-suc);

${ }^{\mathrm{S} 7} \mathrm{PMMA} \mathrm{BaTiO}_{3}$ nanocomposites: Core-shell structured $\mathrm{BaTiO}_{3} /$ poly(methyl methacrylate) (PMMA) nanocomposites;

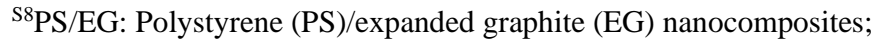

${ }^{\text {S9}}$ rGO-PVA/PVDF: Nanocomposites of poly(vinylidene fluoride) (PVDF) incorporated with reduced graphene oxide (rGO) and poly(vinyl alcohol)-modified rGO (rGO-PVA) ;

${ }^{\mathrm{S} 10} \mathrm{PVA} / \mathrm{V}_{2} \mathrm{C}$ MXene nanocomposite: Water-soluble poly(vinyl alcohol)(PVA)/V ${ }_{2} \mathrm{C}$ MXene nanocomposite films;

${ }^{\mathrm{S} 11} \mathrm{TiO}_{2} / \mathrm{PVP}$ : Poly(4-vinyl phenol) (PVP)/4-hydroxybenzoic acid- $\mathrm{TiO}_{2}$ hybrid. 
Table S4. Tensile properties of $\mathrm{ZrO}_{2}$-PI nanohybrid films.

\begin{tabular}{llll}
\hline Sample & Tensile strength (MPa) & Tensile modulus (MPa) & $\begin{array}{l}\text { Elongation at break } \\
(\%)\end{array}$ \\
\hline PI & $79.7 \pm 6$ & $1627 \pm 175$ & $21.8 \pm 3$ \\
$1 \%$-ZrO 2 -PI & $87.0 \pm 12$ & $2319 \pm 104$ & $15.5 \pm 5$ \\
$3 \%-\mathrm{ZrO}_{2}$-PI & $71.1 \pm 4$ & $2071 \pm 232$ & $11.2 \pm 1$ \\
$5 \%-\mathrm{ZrO}_{2}$-PI & $90.0 \pm 6$ & $2087 \pm 115$ & $9.2 \pm 1$ \\
$7 \%-\mathrm{ZrO}_{2}$-PI & $69.0 \pm 9$ & $2083 \pm 75$ & $6.0 \pm 2$ \\
$10 \%-\mathrm{ZrO}_{2}$-PI & $62.8 \pm 6$ & $2467 \pm 56$ & $5.9 \pm 3$ \\
$20 \%-\mathrm{ZrO}_{2}$-PI & $61.1 \pm 4$ & $2523 \pm 108$ & $5.6 \pm 1$ \\
$30 \%-\mathrm{ZrO}_{2}$-PI & $42.9 \pm 8$ & 3373 & $3.3 \pm 2$ \\
\hline
\end{tabular}

*[Note: The tensile data of the $40 \%-\mathrm{ZrO}_{2}$-PI hybrid film are not available because of their brittle nature.] 
Table S5. Comparison of tensile properties (strength, modulus, elongation at break) and water absorption values of $\mathrm{ZrO}_{2}$-PI nanohybrid films with those of other reported polyimide-based or other polymer-based hybrid films.

\begin{tabular}{|c|c|c|c|c|c|c|}
\hline Samples* & $\begin{array}{l}\text { Filler } \\
\text { Content }\end{array}$ & $\begin{array}{l}\text { Tensile } \\
\text { strength } \\
(\mathrm{MPa})\end{array}$ & $\begin{array}{l}\text { Tensile } \\
\text { modulus } \\
(\mathrm{MPa})\end{array}$ & $\begin{array}{l}\text { Elongation } \\
\text { at break } \\
(\%)\end{array}$ & $\begin{array}{l}\text { Water } \\
\text { Adsorption } \\
(\%)\end{array}$ & References \\
\hline $\mathrm{PI}-\mathrm{ZrO}_{2}$ hybrid films & $0-15$ wt. $\%$ & $N A$ & $N A$ & $N A$ & $2.1-5.9$ & {$[27]$} \\
\hline PI/MS & $0-10$ wt. $\%$ & $42.8-97.2$ & $1770-2367$ & $4.7-22.8$ & $N A$ & {$[36]$} \\
\hline $\begin{array}{l}\mathrm{PI} / \mathrm{ZrO}_{2} \text { composite films } \\
\text { without modifier }\end{array}$ & $0-10$ wt. $\%$ & $N A$ & $N A$ & $N A$ & $1.8-2.3$ & {$[37]$} \\
\hline PI/SiCNWs-GSs & $0-7$ wt. $\%$ & $79.5-95.5$ & $1608-2233$ & $9.6-23.8$ & $N A$ & {$[57]$} \\
\hline $\mathrm{TiO}_{2} / \mathrm{PS}$ & 0-20 wt.\% & $28.5-42$ & $327-520$ & $6.5-11$ & $N A$ & {$[58]$} \\
\hline $\begin{array}{l}\text { f-MWCNTs- } \\
\text { rGO/PMMA }\end{array}$ & $0-3$ wt. $\%$ & $21.2-28.9$ & $1420-2130$ & $N A$ & $N A$ & [59] \\
\hline $\mathrm{PI} / \mathrm{GO}$ & $0-1$ wt. $\%$ & $96-116$ & $2410-3830$ & $N A$ & $1.5-2.6$ & {$[68]$} \\
\hline GFO/pPI & $0-1$ wt. $\%$ & $118-159$ & $2800-4430$ & $14-22$ & $2.2-3.6$ & {$[\mathrm{~S} 2]$} \\
\hline P-POSS-PIs & $0-50$ wt. $\%$ & $86.5-95.7$ & $1210-1330$ & $N A$ & $1.4-2.8$ & {$[\mathrm{~S} 12]$} \\
\hline $\begin{array}{l}\text { Fluorine-contained PI } \\
\text { (FPI) }\end{array}$ & None & $\begin{array}{l}97.2- \\
102.2\end{array}$ & $1190-1680$ & $6-9$ & $1.9-2.5$ & [S13] \\
\hline $\begin{array}{l}\text { Copolyimides } \quad \text { Films } \\
\text { (ODA, BTDA, DAPBI) }\end{array}$ & None & $\begin{array}{l}146.0- \\
220.8\end{array}$ & $3400-6200$ & $6.2-21.5$ & $2.0-3.0$ & [S14] \\
\hline FGO/PI & $0-1$ wt. $\%$ & $\begin{array}{l}93.6- \\
155.7\end{array}$ & $N A$ & $11.1-31.4$ & $1.2-2.4$ & {$[\mathrm{~S} 15]$} \\
\hline $\mathrm{PI} / \mathrm{GO}-\mathrm{NH}_{2}$ & $0-3$ wt. $\%$ & $\begin{array}{l}101.5- \\
156.8\end{array}$ & 1930-3139 & $11.6-6.3$ & $N A$ & {$[\mathrm{~S} 16]$} \\
\hline PI-GO & $0-5$ wt. $\%$ & $51.3-90.6$ & $\begin{array}{l}1331.5- \\
2669.7\end{array}$ & $3.9-22.2$ & $N A$ & {$[S 17]$} \\
\hline h-BN/PVA & $0-40$ vol. $\%$ & $7-17$ & $53-700$ & $7-146$ & $N A$ & {$[\mathrm{~S} 18]$} \\
\hline PVDF/CNTs/Ni@CNTs & $0-10$ wt. $\%$ & $61.3-67.8$ & $510-600$ & $16.9-23.6$ & $N A$ & {$[\mathrm{~S} 19]$} \\
\hline $\begin{array}{l}\mathrm{ZrO}_{2} \text {-PI films derived } \\
\text { from water-soluble PAS }\end{array}$ & $0-40$ wt. $\%$ & $42.9-90.0$ & $1627-3373$ & $3.3-21.8$ & $1.2-3.1$ & This work \\
\hline
\end{tabular}

*[Note: $N A$ represents that this property data is not available; A brief explanation of the above polyimide based hybrid films is summarized as follows].

${ }^{27} \mathrm{PI}-\mathrm{ZrO}_{2}$ hybrid films: (2,2-Bis(3-amino-4-hydroxyphenyl) hexafluoropropane and 4,4-oxydiphthalic anhydride) polyimide /nanocrystalline-zirconium dioxide hybrid materials;

${ }^{36} \mathrm{PI} / \mathrm{MS}$ : Polyimide/mesoporous silica hybrid films based on water-soluble poly(amic acid) ammonium salt;

${ }^{37} \mathrm{PI} / \mathrm{ZrO}_{2}$ composite films without modifier: $\mathrm{ZrO}_{2}$ nanocrystals/Polyimide hybrids; 
${ }^{57} \mathrm{PI} / \mathrm{SiCNW}-\mathrm{GSs}$ : Polyimide (PI) composites containing one-dimensional SiC nanowires grown on twodimensional graphene sheets (1D-2D SiC $\mathrm{NWs}_{\mathrm{s}} \mathrm{GSs}$ ) hybrid fillers;

${ }^{58} \mathrm{TiO}_{2} / \mathrm{PS}:$ Polystyrene/titanium dioxide microcomposites;

${ }^{59}$ f-MWCNTs-rGO/PMMA: Poly(methyl methacrylate) (PMMA) composites containing functionalized multiwalled carbon nanotubes (f-MWCNTs) and reduced graphene oxide (rGO) hybrid nanofillers;

${ }^{68} \mathrm{PI} / \mathrm{GO}$ : Alicyclic polyimide derived from 6FAPB and CBDA /graphene oxide nanocomposites;

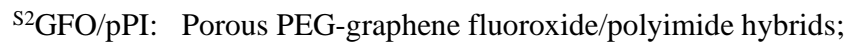

S12P-POSS-PIs: Polyhedral oligomeric silsesquioxanes (POSS) with hierarchical porous structure / PI hybrid film;

${ }^{\text {S13}}$ Fluorine-contained PI (FPI): Sandwich-type porous polyimide film (Fluorine-contained PI (FPI) (6FDA and TFDB));

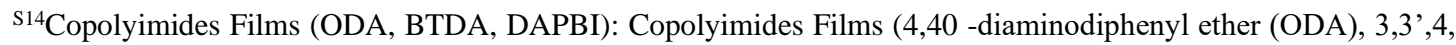
4' -benzophenonetetracarboxylic dianhydride (BTDA), 5,4'-diamino-2-phenyl benzimidazole (DAPBI));

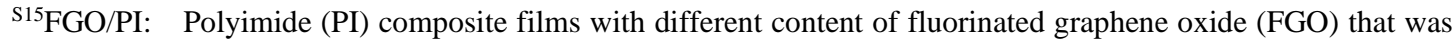
prepared by in situ polymerization;

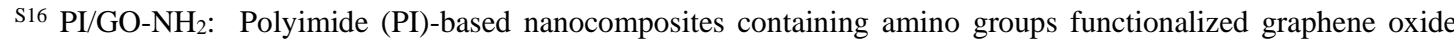
$\left(\mathrm{GO}-\mathrm{NH}_{2}\right)$;

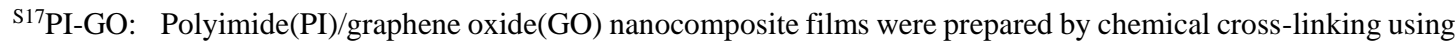
small amounts of divalent $\mathrm{Mg}$ ions;

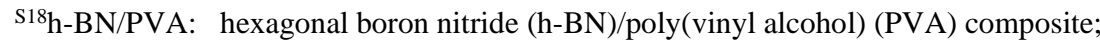

S19PVDF/CNTs/Ni@CNTs: Chain-like Ni@CNTs hybrids are in-situ synthesized by solvothermal method and then the flexible Polyvinylidene fluoride/CNTs/Ni@CNTs composite films are prepared by solution casting and compression molding; 
Table S6. Comparison of permittivity and dielectric loss of $\mathrm{ZrO}_{2}-\mathrm{PI}$ composite films with other reported polyimide-based hybrid films.

\begin{tabular}{|c|c|c|c|c|}
\hline Samples* & Filler Content & Permittivity & Dielectric loss & References \\
\hline CCTO/PI hybrid film $\left(10^{2} \mathrm{~Hz}\right)$ & 40 wt. $\%$ & 49 & 0.2 & [16] \\
\hline $\mathrm{PI}-\mathrm{ZrO}_{2}$ hybrid films $\left(10^{4} \mathrm{~Hz}\right)$ & 10 wt. $\%$ & 6.7 & $N A$ & {$[27]$} \\
\hline $\begin{array}{l}\mathrm{PI} / \mathrm{ZrO}_{2} \text { composite films with } \\
\text { modifier }\left(10^{4} \mathrm{~Hz}\right)\end{array}$ & 10 wt. $\%$ & 3.9 & 0.005 & {$[37]$} \\
\hline hdC-G/PI $\left(10^{2} \mathrm{~Hz}\right)$ & 2.5 wt. $\%$ & 124.9 & 1.97 & {$[60]$} \\
\hline RGO/PI composites $(10 \mathrm{~Hz})$ & $1 \mathrm{wt} . \%$ & 75 & 0.4 & {$[61]$} \\
\hline BT@ $\mathrm{ZrO}_{2} / \mathrm{PI}\left(10^{3} \mathrm{~Hz}\right)$ & 5 wt. $\%$ & 5.5 & 0.02 & {$[64]$} \\
\hline K-HNTs/PI $\left(10^{2} \mathrm{~Hz}\right)$ & 20 wt. $\%$ & 4.0 & 0.02 & {$[\mathrm{~S} 20]$} \\
\hline BT@PDA/PI (10² Hz) & 7 wt.\% & 7.7 & 0.043 & {$[\mathrm{~S} 21]$} \\
\hline $\mathrm{Ag}-\mathrm{ND} / \mathrm{PI}\left(10^{3} \mathrm{~Hz}\right)$ & $1 \mathrm{wt} . \%$ & 7.8 & 0.025 & {$[\mathrm{~S} 22]$} \\
\hline $\begin{array}{l}\mathrm{PI} / \mathrm{ZrO}_{2} \text {-nanocrystal hybrid film } \\
\left(10^{2} \mathrm{~Hz}\right)\end{array}$ & 33 wt. $\%$ & 4.7 & $N A$ & {$[\mathrm{~S} 23]$} \\
\hline $10 \%-\mathrm{ZrO}_{2}-\mathrm{PI}\left(10-10^{4} \mathrm{~Hz}\right)$ & 10 wt. $\%$ & $4.5-5.1$ & $0.012-0.047$ & This work \\
\hline $20 \%-\mathrm{ZrO}_{2}-\mathrm{PI}\left(10-10^{4} \mathrm{~Hz}\right)$ & 20 wt. $\%$ & $4.5-5.2$ & $0.012-0.060$ & This work \\
\hline $30 \%-\mathrm{ZrO}_{2}-\mathrm{PI}\left(10-10^{4} \mathrm{~Hz}\right)$ & 30 wt. $\%$ & $4.6-5.9$ & $0.022-0.133$ & This work \\
\hline $40 \%-\mathrm{ZrO}_{2}-\mathrm{PI}\left(10-10^{4} \mathrm{~Hz}\right)$ & 40 wt. $\%$ & $4.6-6.2$ & $0.030-0.163$ & This work \\
\hline
\end{tabular}

*[Note: $N A$ represents that this property data is not available; A brief explanation of the above polyimide based hybrid films is summarized as follows].

${ }^{16} \mathrm{CCTO} / \mathrm{PI}$ hybrid film: Calcium copper titanate/Polyimide hybrid films;

${ }^{27} \mathrm{PI}-\mathrm{ZrO}_{2}$ hybrid films: Polyimide(2,2-bis(3-amino-4-hydroxyphenyl) hexafluoropropane and 4,4-oxydiphthalic anhydride)/nanocrystalline-zirconium dioxide hybrid materials;

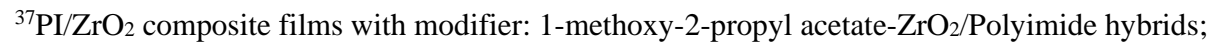

${ }^{60} \mathrm{hdC}-\mathrm{G} / \mathrm{PI}$ : Homogeneous dispersion of CNTs and graphene oxide (hdC-G) via solvent-exchange, followed by fabricating hdC-G/polyimide (PI) hybrid films by in situ polymerization and thermal imidization.

${ }^{61}$ RGO/PI composites: Controllable reduction of graphene oxide(RGO)/Polyimide hybrids;

${ }^{64} \mathrm{BT} @ \mathrm{ZrO}_{2} / \mathrm{PI}: \quad$ 1-dimensional core-shell structured $\mathrm{BaTiO}_{3} @ \mathrm{ZrO}_{2} \quad\left(\mathrm{BT} @ \mathrm{ZrO}_{2}\right)$ nanofibers as filler and polyimide $(\mathrm{PI})$ as the polymer matrix;

${ }^{\mathrm{S} 20} \mathrm{~K}-\mathrm{HNTs} / \mathrm{PI}$ : 3-aminopropyl triethoxysilane modified HNTs (K-HNTs)/polyimide hybrids;

S21BT@PDA/PI: Polyimide (PI) nanocomposite films containing barium titanate@ @olydopamine (BT@PDA) nanoparticles within a core-shell structure ;

S22Ag-ND/PI: Ag nanodot/polyimide (Ag-ND/PI) nanocomposite films;

${ }^{\mathrm{S} 23} \mathrm{PI} / \mathrm{ZrO}_{2}$-nanocrystal hybrid film: Oleic acid-capped $\mathrm{ZrO}_{2}$ nanocrystals/Polyimide hybrids; 


\section{REFERENCES}

(S1) Deng, W.; Ren, G. G.; Wang, W. Q.; Cui, W. W.; Luo, W. J. Enhanced dielectric properties and thermostability in polyimide composites with core-shell structured polyimide@ $\mathrm{BaTiO}_{3}$ nanoparticles. High Perform Polym 2021, 0954008321993526. https://doi.org/10.1177/0954008321993526.

(S2) Chen, Z. G.; Liu, S. M.; Yan, S. J.; Shu, X.; Yuan, Y. C.; Huang, H. H.; Zhao, J. Q. Overall improvement in dielectric and mechanical properties of porous graphene fluoroxide/polyimide nanocomposite films via bubblestretching approach. Mater. Des. 2017, 117, 150-156.

(S3) Tsai, M. H.; Tseng, I. H.; Chiang, J. C.; Li, J. J. Flexible Polyimide Films Hybrid with Functionalized Boron Nitride and Graphene Oxide Simultaneously To Improve Thermal Conduction and Dimensional Stability. ACS Appl. Mater. Interfaces 2014, 6, 8639-8645.

(S4) Huang, T. T.; Tsai, C. L.; Tateyama, S. J.; Kaneko, T.; Liou, G. S. Highly transparent and flexible bio-based polyimide $/ \mathrm{TiO}_{2}$ and $\mathrm{ZrO}_{2}$ hybrid films with tunable refractive index, Abbe number, and memory properties. Nanoscale 2016, 8, 12793-12802.

(S5) Tsai, C. L.; Lee, T. M.; Liou, G. S. Novel solution-processable functional polyimide/ZrO $/ \mathrm{Z}_{2}$ hybrids with tunable digital memory behaviors. Polym. Chem. 2016, 7, 4873.

(S6) Li, T. L.; Hsu, S. L. C. Enhanced Thermal Conductivity of Polyimide Films via a Hybrid of Micro- and NanoSized Boron Nitride. J. Phys. Chem. B 2010, 114, 6825-6829.

(S7) Xie, L. Y.; Huang, X. Y.; Wu, C.; Jiang, P. K. Core-shell structured poly(methyl methacrylate)/BaTiO 3 nanocomposites prepared by in situ atom transfer radical polymerization: a route to high dielectric constant materials with the inherent low loss of the base polymer. J. Mater. Chem. 2011, 21, 5897.

(S8) Goyal, R. K.; Jagadale, P. A.; Mulik, U. P. Thermal, Mechanical, and Dielectric Properties of Polystyrene/Expanded Graphite Nanocomposites. J. Appl. Polym. Sci. 2009, 111(4), 2071-2077.

(S9) Wang, D. R.; Bao, Y. R.; Zha, J. W.; Zhao, J.; Dang, Z. M.; Hu, G. H. Improved Dielectric Properties of Nanocomposites Based on Poly(vinylidene fluoride) and Poly(vinyl alcohol)-Functionalized Graphene. ACS Appl. Mater. Interfaces 2012, 4, 6273-6279.

(S10) Deng, Q. H.; Zhou, F. R.; Qin, B.; Feng, Y. F.; Xu, Z. C. Eco-friendly poly(vinyl alcohol)/delaminated $\mathrm{V}_{2} \mathrm{C}$ MXene high-knanocomposites with low dielectric loss enabled by moderate polarizationand charge density at the interface. Ceram. Int. 2020, 46, 27326-27335.

(S11) Kim, Y. J.; Kim, J. H.; Kim, Y. S.; Lee, J. K. TiO2-poly(4-vinylphenol) nanocomposite dielectrics for organic thin film transistors. Org. Electron. 2013, 14, 3406-3414.

(S12) He, Z.; Xie, J. W.; Liao, Z. W.; Ma, Y. Y.; Zhang, M. Y.; Zhang, W. L.; Yue, H. Y.; Ggao, X. Hierarchical porous structure contained composite polyimide film with enhanced dielectric and water resistance properties for dielectric material. Prog. Org. Coat. 2021, 151, 106030. 
(S13) Xu, L.; Ma, Y. Y.; Xie, J. W.; Zhang, W. L.; Wu, Z. J.; He, Z. F. Sandwich-type porous polyimide film with improved dielectric, water resistance and mechanical properties. J. Mater. Sci. 2019, 54, 5952-5960.

(S14) Yu, X. H.; Liang, W. H.; Cao, J. H.; Wu, D. Y. Mixed Rigid and Flexible Component Design for HighPerformance Polyimide Films. Polymers 2017, 9, 451.

(S15) He, D. Q.; Wang, Z. G.; Long, J. P.; Liang, B. Preparation of Fluorinated Graphene Oxide/Polyimide Composites with Low Dielectric Constant and Moisture Resistance. Nano 2018, 13(08), 1850098.

(S16) Wang, C. Y.; Lan, Y. F.; Yu, W. T.; Li, X.; Qian, Y.; Liu, H. S. Preparation of amino-functionalized graphene oxide/polyimidecomposite films with improved mechanical, thermal and hydrophobicproperties. Appl. Surf. Sci. 2016, 362, 11-19.

(S17) Kong, J. Y.; Choi, M. C.; Kim, G. Y; Park, J. J.; Selvaraj, M.; Han, M. J.; Ha, C. S. Preparation and properties of polyimide/graphene oxide nanocomposite films with Mg ion crosslinker. Eur. Polym. J. 2012, 48, 1394-1405.

(S18) Zhang, J.; Wang, X. N.; Yu, C. P.; Li, Q. L.; Li, Z.; Li, C. W.; Lu, H. F.; Zhang, Q. C.; Zhao, J. X.; Hu, M.; Yao, Y. G. A facile method to prepare flexible boron nitride/poly(vinyl alcohol) composites with enhanced thermal conductivity. Compos Sci Technol 2017, 149, 41-47.

(S19) Zeng, S. P.; Li, X. P.; Li, M. J.; Zheng, J. J.; E, S. J.; Yang, W. J.; Zhao, B.; Guo, X. Q.; Zhang, R. Flexible PVDF/CNTs/Ni@CNTs composite films possessing excellent electromagnetic interference shielding and mechanical properties under heat treatment. Carbon 2019, 155, 34-43.

(S20) Zhu, T. W.; Qian, C.; Zheng, W. W.; Bei, R. X.; Liu, S. W.; Chi, Z. G.; Chen, X. D.; Zhang, Y.; Xu, J. R. Modified halloysite nanotube filled polyimide composites for film capacitors: high dielectric constant, low dielectric loss and excellent heat resistance. RSC Adv. 2018, 8, 10522-10531.

(S21) Wu, Z. Q; Zhou, H. H.; Guo, Q.; Liu, Z. G.; Gong, L.; Zhang, Q. Y.; Zhong, G. J.; Li, Z. M.; Chen, Y. H. Enhanced dielectric properties in polyimide nanocomposites containing barium titanate@ polydopamine coreshell nanoparticles. J. Alloys Compd. 2020, 845, 156171.

(S22) Xing, S.; Pan, Z. B.; Wu, X. F.; Chen, H. X.; Lv, X. J.; Li, P.; Liu, J. J.; Zhai, J. W. Enhancement of thermal stability and energy storage capability of flexible Ag nanodot/polyimide nanocomposite films via in situ synthesis. J. Mater. Chem. C 2020, 8(36), 12607-12614.

(S23) Li, X. T.; Wang, G.; Huang, L. J.; Kang, X. J.; Cheng, F. M.; Zhao, W. J.; Li, H. D. Significant enhancement in dielectric constant of polyimide thin films by doping zirconia nanocrystals. Mater. Lett. 2015, 148, 22-25. 